Supporting Information

\title{
Tailoring Material Stiffness by Filler Particle
}

\section{Organization}

Peiying J. Tsai, ${ }^{1}$ Suvojit Ghosh,$^{2}$ Peidong $W u,{ }^{1}$ and Ishwar K. Puri ${ }^{1,2 *}$

${ }^{1}$ Department of Mechanical Engineering, ${ }^{2}$ Department of Engineering Physics, McMaster

University, 1280 Main Street West, Hamilton, Ontario, L8S 4L7, Canada

*Email: ikpuri@mcmaster.ca 
Applying the appropriate boundary conditions is essential when using unit cell methods or representative volume elements. In the case when the repetitive configuration shows symmetry, such as in Fig. 1a, b, and d, the modeled volume is reduced to $1 / 8$ of the repetitive cell by applying the boundary conditions that account for symmetry. ${ }^{1-2}$ For uniaxial tension in $y$ direction,

$$
\begin{array}{ll}
\left.u\right|_{x=0}=0, & \left.u\right|_{x=b_{x}}=b_{x} \varepsilon_{x}=U, \\
\left.v\right|_{y=0}=0, & \left.v\right|_{y=b_{y}}=b_{y} \varepsilon_{y}^{0}=V, \\
\left.w\right|_{z=0}=0, & \left.w\right|_{z=b_{z}}=b_{z} \varepsilon_{z}=W,
\end{array}
$$

where $u, v$, and $w$ denote displacements along the $x, y$, and $z$ directions, $b_{j}$ the dimension of the unit cell, $\left.\right|_{k}$ the corresponding quantity at surface $k$, and $\varepsilon_{y}^{0}$ the prescribed macroscopic normal strain (while $\varepsilon_{x}$ and $\varepsilon_{z}$ are determined during simulations through the displacements $U$ and $W)$.

In ABAQUS ${ }^{\mathrm{TM}}$, the symmetric boundary conditions, $u=U_{1}=0, v=U_{2}=0$, and $w=U_{3}=0$ , are applied on the symmetric faces $x=0, y=0$, and $z=0$, respectively. Equation constraints are implemented on the opposite surfaces using a reference control point for each surface, as

$$
\begin{aligned}
& X(\text { dof: } 1)-C_{x}(\text { dof: } 1)=0, \\
& Y(\text { dof: } 2)-C_{y}(\text { dof: } 2)=0, \\
& Z(\text { dof: } 3)-C_{z}(\text { dof: } 3)=0,
\end{aligned}
$$

where $X, Y$, and $Z$ denote sets of nodes on the corresponding surfaces, and $C_{x}, C_{y}$, and $C_{z}$ are node sets containing only a single reference point within each set to control the degree of freedom (dof), where dof:1,2, and 3 are displacements along the $x, y$, and $z$ directions. A displacement boundary condition is set on $C_{y}$ as the prescribed uniaxial tensile strain through $b_{y} \varepsilon_{y}^{0}=V$. No 
boundary conditions are applied on $C_{x}$ and $C_{z}$. By using the control point and the equation constraints, the surfaces remain parallel to their initial states after deformation. 


\section{REFERENCES}

(1) Li, S., Boundary Conditions for Unit Cells from Periodic Microstructures and Their Implications. Compos. Sci. Technol. 2008, 68, 1962-1974.

(2) Li, S.; Wongsto, A., Unit Cells for Micromechanical Analyses of Particle-Reinforced Composites. Mech. Mater. 2004, 36, 543-572. 